\title{
Correction to: Cell dynamics during differentiation therapy with all- trans retinoic acid in acute promyelocytic leukemia
}

\author{
Kazuyuki Sato ${ }^{1}$. Hirotaka Sakai ${ }^{1}$ - Yusuke Saiki ${ }^{1}$. Akiko Uchida ${ }^{1} \cdot$ Yu Uemura ${ }^{1}$. Satoshi Yokoi ${ }^{1} \cdot$ Yuka Tsuruoka $^{1}$. \\ Yuji Nishio ${ }^{1}$. Manabu Matsunawa ${ }^{1}$ - Yoshinori Suzuki ${ }^{1}$ 'Yasushi Isobe ${ }^{1} \cdot$ Masayuki Kato $^{1} \cdot$ Naoto Tomita $^{1}$. \\ Yasuyuki Inoue ${ }^{1} \cdot$ Ikuo Miura $^{1}$
}

Published online: 12 August 2018

(c) The Japanese Society of Hematology 2018

\section{Correction to: International Journal of Hematology https://doi.org/10.1007/s12185-018-2472-9}

In the original publication of the article, Table 2 was published incorrectly. The column names were swapped under the column heading "Prom (\%)". The correct column names are $\mathrm{PB}$ and $\mathrm{BM}$.

The original article can be found online at https://doi.org/10.1007/ s12185-018-2472-9.

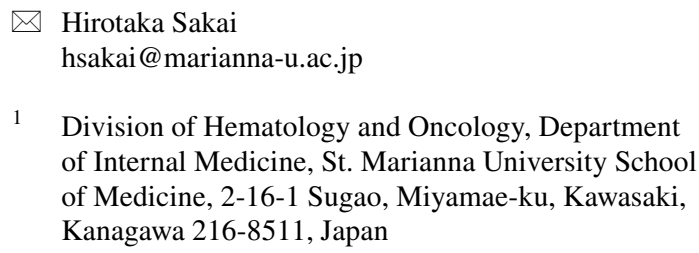

1 Division of Hematology and Oncology, Department of Internal Medicine, St. Marianna University School of Medicine, 2-16-1 Sugao, Miyamae-ku, Kawasaki, Kanagawa 216-8511, Japan 